\title{
Endosonography-guided fine-needle aspiration biopsy
}

in differential diagnosis of adrenal gland tumors - pilot study.

Michal Krcma', Vaclav Hejda', Eva Dvorakova

${ }^{1}$ Endocrinology, $1^{\text {st }}$ Dept. of Clinic Medicine, Teaching Hospital and Charles University Plzen, Czech Republic

${ }^{2}$ Gastroenterology, $1^{\text {st }}$ Dept. of Clinic Medicine, Teaching Hospital and Charles University Plzen, Czech Republic

\section{N T R O D U C T I O N}

Adrenal incidentaloma is a problem with increasing importance. There are CT-density and washout criteria for distinction between adrenal adenoma and other tumors, there are well established protocol of screening for malignancy and/or hormonal dysfunction. But there remains small portion of atypical adenoma where detailed diagnostic is needed. One relatively new option is endosonographyguided fine-needle aspiration biopsy.

Aim: To assess validity, complications and diagnostic contribution of endosonography-guided fine-needle aspiration biopsy in patients with atypical adrenal tumor.

\section{I A G NOS T I C A L G O R I T H M}

1) Overall malignancy screening: physical examination, chest $X$ ray, prostatic specific antigen (men), gynecology examination including transvaginal ultrasonography (women), faecal occult blood detection or primary screening colonoscopy

2) CT criteria: size ( $>4 \mathrm{~cm})$, density (washout), homogenity

3) hormonal screening: common biochemistry, morning cortisol and low dose $(1 \mathrm{mg}$ ) dexamethasone suppression test, aldosterone/renin ratio, plasmatic metanephrines, (optionally dehydroepiandrostendion sulphate)

W H A T M A N $S$ "ATYPICAL" TUMOR? hormonally inactive

slow washout and /or native density above $40 \mathrm{HU}$

non-homogenous structure on CT scan

Part of atypical adrenal tumors are lipid-poor adenoma, and therefore is important to distinguish between this type of adenoma and metastasis or adrenal carcinoma.

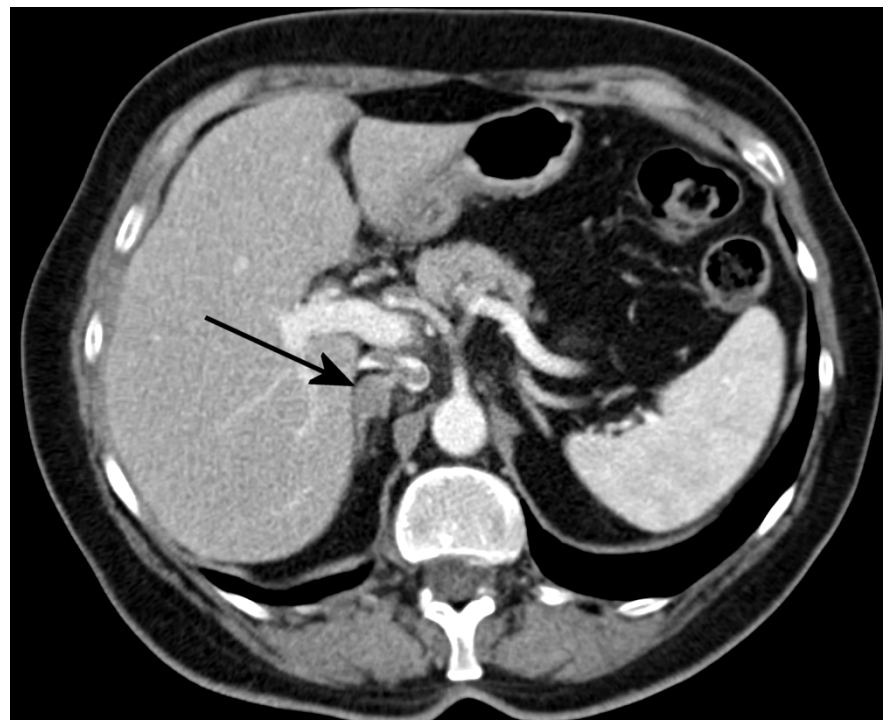

P A T I E N T S A N D M E T HO D S

14 patients ( 6 men, 8 women) with atypical adrenal mass were selected from for the study. In all patients was performed common screening for malignancy and/or hormonal dysfunction. Excluding criteria was typical adrenal adenoma and functional adrenal tumor. In all patients were examined plasmatic metanephrines and was excluded feochromocytoma before biopsy. All patients were admitted after endosonographic biopsy to clinical department and were observed till next day. In all patients were not observed any complication of biopsy.

In 5 cases there were bilateral tumours, in 3 cases right adrenal tumours and in 6 cases left adrenal tumor. 3 tumours were bigger than $4 \mathrm{~cm}$; others were smaller than $4 \mathrm{~cm}$.

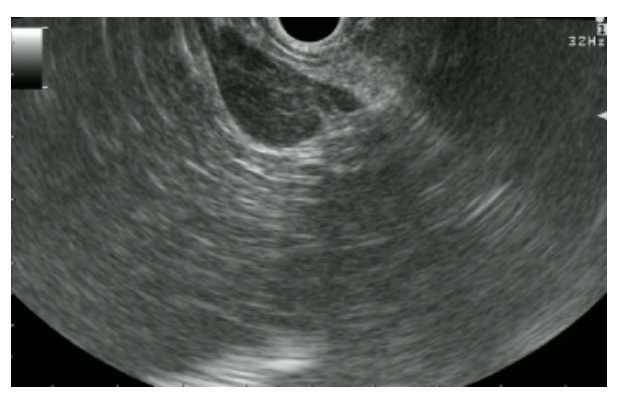

Endosongraphic imaging of adrenal gland - this was adenoma $<4 \mathrm{~cm}$

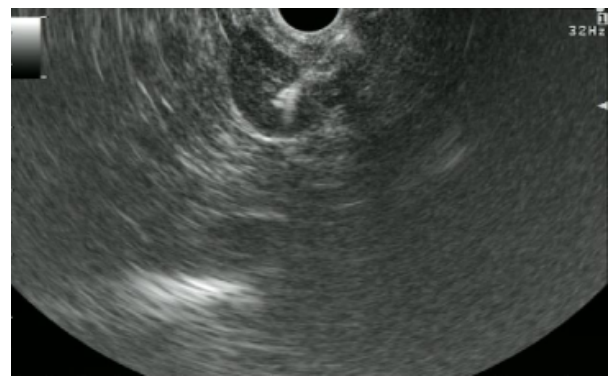

Adrenal biopsy during endo-sonography (white line is a needle)

\section{R E S U L T S}

In one case of unilateral tumor no biopsy was performed - adrenal gland was too small for secure biopsy; in one case of bilateral tumor was performed only one sample from anatomical reasons.

17 samples in 14 patients; 2 marginally sufficient; 15 sufficient

\begin{tabular}{|l|l|l|}
\hline $\begin{array}{l}5 \text { cases - resulted } \\
\text { to adrenalectomy }\end{array}$ & \multicolumn{1}{|c|}{ biopsy } & histology from surgery \\
\hline & $1 x$ feochromocytoma & feochromocytoma \\
\hline & $1 \times$ suspected NET & $\begin{array}{l}\text { metastasis of ovarian NET } \\
\text { (patient was after } \\
\text { hysterectomy) }\end{array}$ \\
\hline & $3 x$ adrenal malignancy & $\begin{array}{l}\text { 2x adrenal carcinoma } \\
1 x \text { adrenal cortex tumor } \\
\text { with borderline } \\
\text { characteristic between } \\
\text { adenoma and carcinoma }\end{array}$ \\
\hline
\end{tabular}

9 cases - observation, in 6 from this cases after $6 \mathrm{M}$ were no progression or other malignancy, 3 cases are very recent.

\section{O N C L U S I O N}

Endosonography-guided fine-needle aspiration biopsy seems to be useful and safe method of differential diagnosis of atypical adrenal tumours. It can reveal any primary or secondary malignancy in early stage, with good possibility of surgical treatment.

Small number of patients makes statistic analysis impossible.

krcmam@fnplzen.cz 